\title{
A Spatio-Temporal Analysis of Dengue Spread in a Brazilian Dry Climate Region
}

\section{Aloísio Nascimento Filho ( $\square$ aloisio.nascimento@gmail.com )}

Serviço Nacional de Aprendizagem Industrial

\section{Thiago Murari}

Serviço Nacional de Aprendizagem Industrial

\section{Paulo Ferreira}

Polytechnic Institute of Portalegre

\section{Hugo Saba}

Serviço Nacional de Aprendizagem Industrial

\section{Marcelo Moret}

Serviço Nacional de Aprendizagem Industrial

\section{Research Article}

Keywords: dengue, regions, Salvador

Posted Date: November 25th, 2020

DOI: https://doi.org/10.21203/rs.3.rs-110616/v1

License: (c) (1) This work is licensed under a Creative Commons Attribution 4.0 International License. Read Full License 


\title{
A spatio-temporal analysis of dengue spread in a Brazilian dry climate region
}

\author{
Aloísio .S. Nascimento Filho ${ }^{1, *}$, Thiago B. Murari ${ }^{1,+}$, Paulo Ferreira ${ }^{2,3,4,+}$, Hugo Saba ${ }^{1,5,+}$, \\ and Marcelo A. Moret ${ }^{1,5,+}$
}

${ }^{1}$ Centro Universitário Senai Cimatec - Salvador, Bahia, Brazil

${ }^{2}$ VALORIZA - Research Center for Endogenous Resources Valorization, Portalegre, Portugal

${ }^{3}$ Instituto Politécnico de Portalegre, Portalegre, Portugal

${ }^{4}$ CEFAGE-UE, IIFA, Universidade de Ev́ora, Ev́ora, Portugal

${ }^{5}$ Universidade do Estado da Bahia, Salvador, BA, Brazil

*corresponding autthor.aloisio.nascimento@gmail.com

+these authors contributed equally to this work

\begin{abstract}
We investigated the relation between the spread, time scale, and spatial arrangement of dengue in Bahia, a Brazilian dry climate region, for the period 2000 to 2009. The degree of cross-correlation is calculated for 15 economic regions. We apply a multiscale statistical analysis to datasets of dengue cases in order to verify the effect of infection dispersal on the economic regions from the metropolitan region of Salvador. Our empirical results support a significant and persistent cross-correlation between most economic regions, reinforcing the idea that there are no economic, environmental or climatic barriers to the spread of dengue. Our main contribution lies in the cross-correlation results revealing multiple aspects related to the propagation of dengue for dry climate regions.
\end{abstract}

\section{Introduction}

Dengue, Zika and Chikungunya are caused by arboviruses that are transmitted in urban centers by the same arthropod vector, Aedes aegypti mosquitos (AA). Once infected, the mosquito can transmit the virus during her whole lifetime. However, the course of an infection differs between people. The transmission of these arboviruses is complex, involving the various aspects of the behavior of the vectors as well as of the infected humans ${ }^{1}$. To study the tracking and history of a disease had been a way to understanding its behavior ${ }^{2}$. We believe that evaluating temporal interactions of case records may reveal relevant clues about the disease spread process, mainly in large areas. For instance, large cities can be offer to provide favorable conditions for arboviruses transmission due to the ease circulation of the infected people.

Spatial analysis has been able to construct scenarios and identifying health impacts ${ }^{3}$. Studies involving spatial and temporal disease have tried to identify the dengue infection spread patterns. For instance, spatial and temporal patterns of dengue infection were studied in two cities in Taiwan by applying cluster analysis and two distinct diffusion behaviors were found. A rapid dispersal and a relocation pattern of temporal movement involving clusters ${ }^{4}$. A study involving pattern of dengue and hemorrhagic dengue incidence in several districts were applied within the Songkhla municipality in Thailand. The founds reveal that the incidence was strongly associated with the percentages of shop-houses, brick-built houses and houses with poor garbage disposal ${ }^{5}$.

Brazil has a large territory with significant vulnerable to arboviruses. In the last years Zika and Chikungunya joined dengue and became relevant Brazilian public health problems. Thus, once Zika and Chikungunya data are still scarce, by using dengue data may provide important clues about their spread patters. Bahia is the fourth largest Brazilian population, with 14,016,906 inhabitants, Bahia, inside of $564.732 \mathrm{~km}^{2}$, an extensive road network interconnecting all municipalities in the state ${ }^{6}$. The transportation network in Bahia is linked by 22 federal highway and 11 state highways covered all 417 Bahia's municipalities ${ }^{7}$. Once in Bahia the AA is present in $99.5 \%$ of municipalities, combating the spread of arboviruses is a challenge for public health.

Besides, Bahia has specific climatic conditions such as low precipitation, as shown in Figure 1. This may justify the non-homogeneous low number of cases of arboviruses infections, because climate is usually associated with such problems. Nevertheless, according to ${ }^{1}$ high levels of precipitation and a temperature suitable for dengue transmission are strongly associated with elevated dengue risk, although low precipitation was not found to limit transmission strongly. 


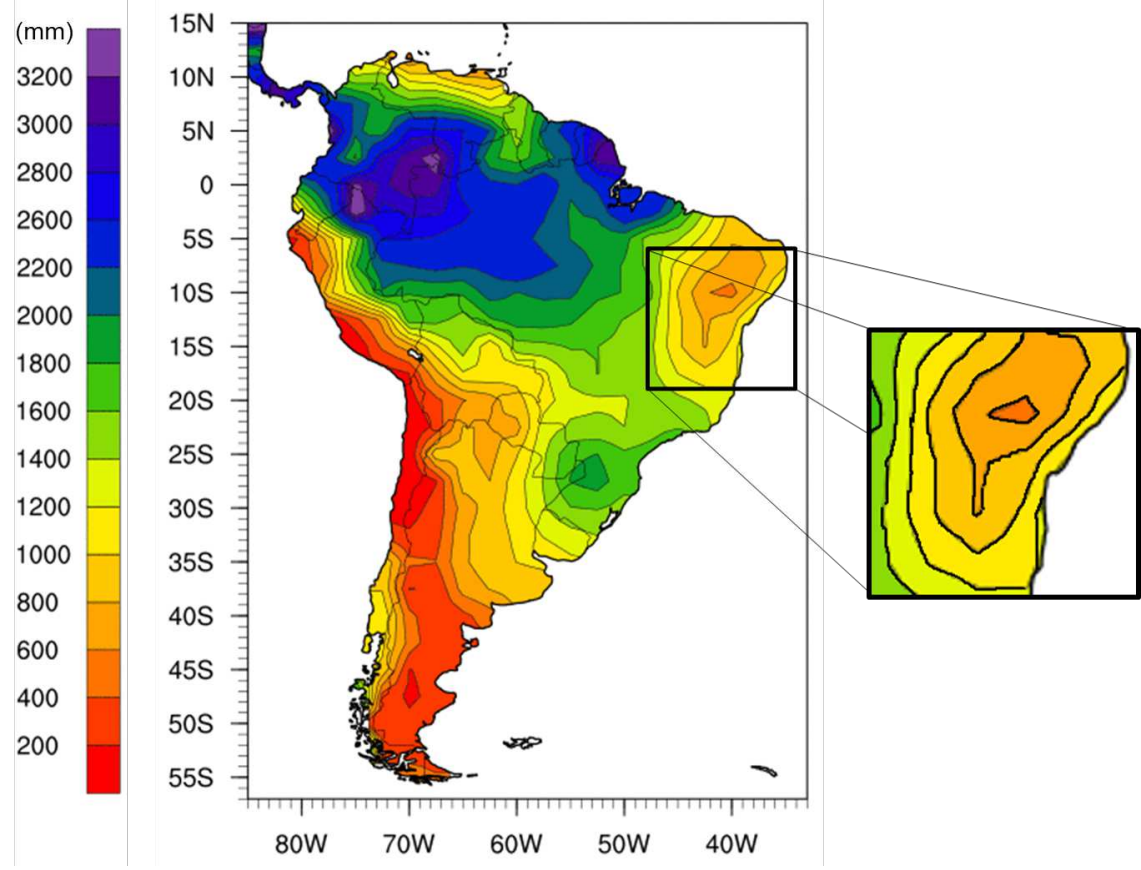

Figure 1. The annual total precipitation in Bahia. According to the Physical Science Division of U.S. department of commerce between 1976 and 2009 this was less than 1,000 mm. Figure adapted from ${ }^{8}$

The study that measured the existence of a spatial correlation between socioeconomic, demographic, environmental variables and the incidence of dengue in a medium-sized city in Brazil, found spatial dependence of the incidence of dengue, socioeconomic factors and the organization of urban space ${ }^{9}$. For insentience, the relevance in the diffusion of dengue across the state of Bahia-Brazil was observed by applying correlations between occurrences of reported cases of dengue among municipalities ${ }^{2,7,10}$. Another study evaluated whether the spread of dengue fever can be explained by differences in regional economies, concluding that this disease was not influenced by economic aspects or regional arrangement, and also suggesting that the disease's AA vector has adapted to all economic regions ${ }^{11}$.

Once there are not effective chemical and biological vector control, and the fact that no vaccine against dengue, Zika and Chikungunya are not yet available, the hypothesis of the pathways and speed of arbovirus circulation in Bahia-Brazil may be relevant factors to interrupt arboviruses spread. Taking into account that time series of dengue are available, we propose to evaluate the speed and dynamics of the spread of disease. For this purpose we verify the pathways of dengue by applying cross-correlation DCCA statistical method in dengue cases Bahia-Brazil between 2000 and 2009.

\section{Results}

We calculated 105 pairs for 29 different time scales, considering boxes from 4 to 362 days. Figure 2 shows the cross-correlations between RMS with all ER. The following regions presented a strong cross-correlation coefficient after 33 days: LTN, REC and PGU. A cross-correlation is considered strong when the $\rho D C C A$ is greater than $0.66^{12}$. All the other regions did not achieve the strong cross-correlation coefficient, but they are statistically significant, except IRC and BMF that become non significant after 154 days.

The pathways of the dengue spread are shown on Figure 3. Only for a better visualization of this spread, we proposed a classification for the coefficient degree, as follow: Strong: $\rho_{D C C A}>0.66$, Medium: $0.4<\rho_{D C C A} \leq 0.66$, Weak: $0.2<\rho_{D C C A} \leq 0.4$, and Very weak: $\rho_{D C C A} \leq 0.2$. It shows the maintenance of the strong correlation for ER around RMS borders for any time scale. Between 154 and 254 days, there is a weak to medium coefficient movement in almost all ER of Bahia that do not border with RMS, except for EXS, SGE, BMF and IRC. The spread remains unchanged after 254 days. This behavior may suggest that the spread is straight associated to the distance between municipalities. 


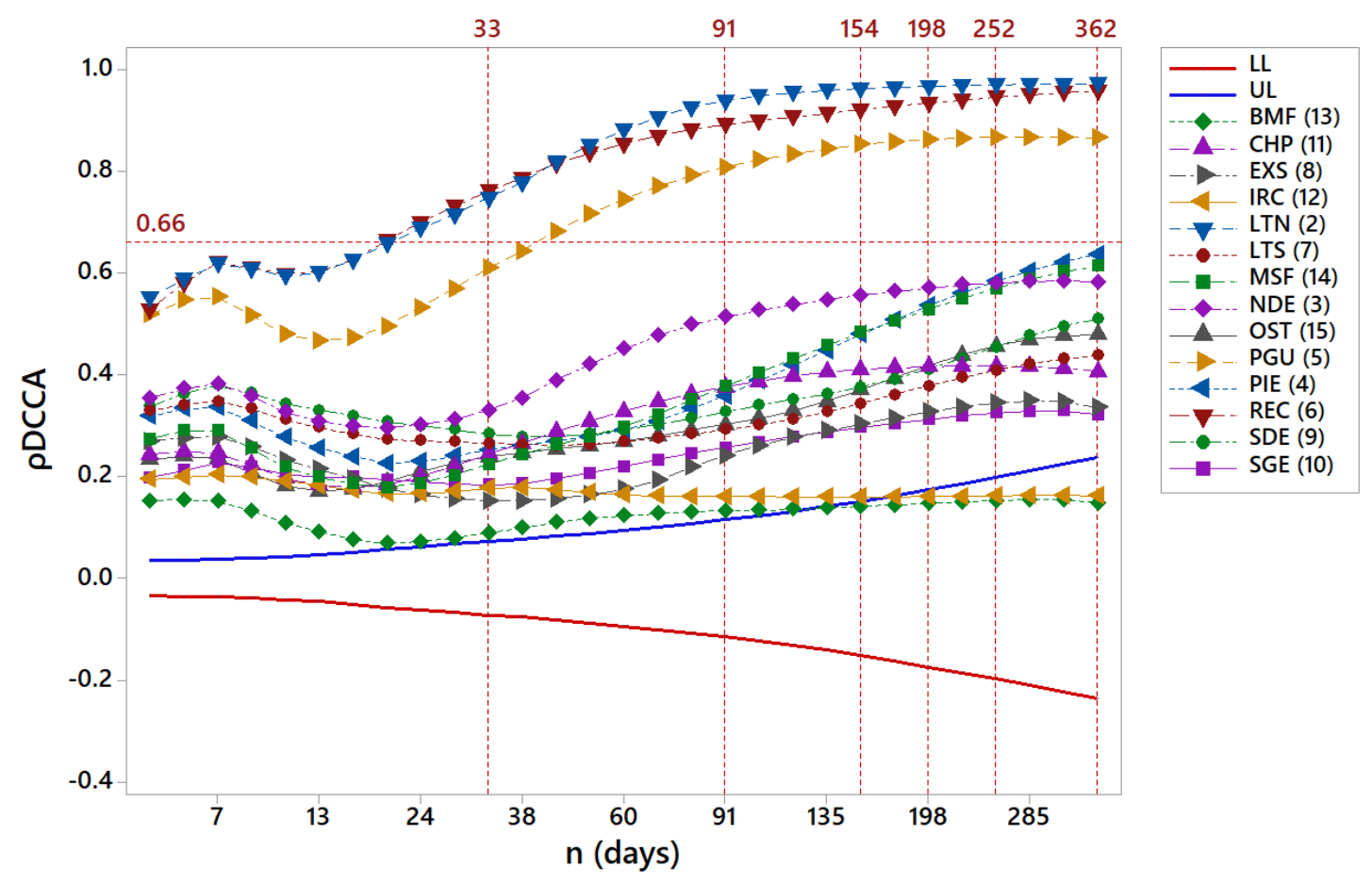

Figure 2. Cross-correlation results for 29 different time scales. The values for $\rho_{D C C A}$ between RMS and the nearby ER presented a strong significant correlation. LTN, REC and PGU stood out by exceeding the correlation level of 0.66, from the scale of 33 days, remaining strong for all subsequent time scales. 95\%LL and 95\% UL represent the lower and upper limits to consider for the test of the existence or not of significant correlation

This dengue spread behavior seems to be related to the direct links between RMS and other ER, represented in figure 5 . Direct links are located mostly in the border regions of RMS. The exceptions are BMF and IRC, that have direct links but are on the very weak range of the coefficient degree. These two ER are within the lowest precipitation area of the analyzed region as can be observed in Figure 1.

\section{Discussion}

The results suggest that the arboviruses spread is straight associated to the distance between ER in the dry climate region. The distance impacts on the coefficient degree along the time. The proximity to low-income urban and peri-urban centres was also linked to greater risk, particularly in highly connected areas, indicating that human movement between population centres is an important facilitator of dengue spread ${ }^{10}$.

Besides climate features, it was possible to observe the interaction of distinct economic arrangement in Bahia, with apparent interconnection having the capacity to compose the transmission chain, and hence the spatio-temporal spread of the virus. The multiscale analysis for dengue case infections allows an understanding of the spread taking into account different time scales. By studying the correlation between ER, it was possible to compare the virus spread trajectory.

The statistic method, multiscale $\rho_{D C C A}$ cross correlation applied to the different dengue cases arrangements measured the degree of correlation from RMS. The remarkable increase of degree coefficient allowed determinate the time scale dynamic of the dengue spread in a Brazilian dry region, where in the begging the $\rho_{D C C A}$ coefficient becomes strong after 33 days for RMS board ER. We believe that approach has the advantage of setting aside local and regional details, such as economic aggregates, areas and distances. By offering an opportunity to evaluate the dengue propagation pathways and its dynamic. The empirical results suggest that cities with role of territorial management and high levels of centrality as Salvador in RMS or cities situated in REC, as well as intensity of the connections between cities play a critical role in the spreading process. Where that may 


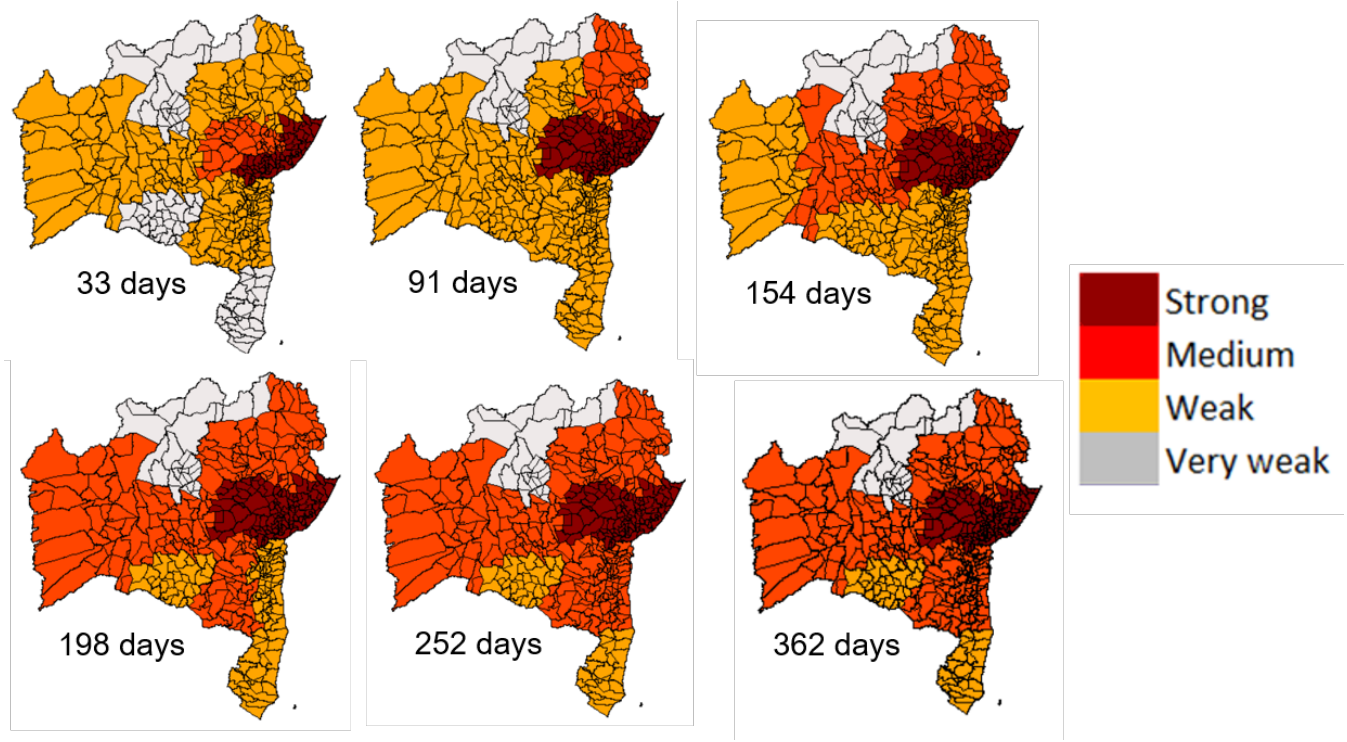

Figure 3. Correlation degree between RMS and all economic regions in Bahia-Brazil for different time scales. The value of the $\rho_{D C C A}$ between RMS and all ER presented a significant correlation. This was calculated for six scale days, 33, 91, 154, 198, 252 and 362 days. The $\rho_{D C C A}$ 's ranges were associated with colors (Strong: $\rho_{D C C A}>0.66$, Medium: $0.4<\rho_{D C C A} \leq 0.66$, Weak: $0.2<\rho_{D C C A} \leq 0.4$, and Very weak: $\left.\rho_{D C C A} \leq 0.2\right)$ in order assist understanding.

providing favourable conditions dengue propagation. Thus, these regions should have their own set of actions to combat dengue prioritizing to interrupt dengue propagation, still in the beginning, approximately 30 days to interrupt a possible outbreak process.

\section{Methods}

Daily records of dengue cases in Bahia were collected between 2000 and 2009 from the Brazilian Diseases Notification System databases from the Brazilian health ministry ${ }^{13}$. The data were aggregated into economic regions (ER), as proposed by ${ }^{14}$, and in Figure 4 a map to assist understanding.

The most important ER (RMS) was chosen in this analysis due to economic importance and to include the capital of Bahia, Salvador. Furthermore, Salvador is classified as Influence Area of the City (IAC) ${ }^{6}$ as showed in Figure 5. IAC are characterized by include intensity of the connections between cities, plays a role of territorial management, evaluating levels of centrality of the executive and judiciary at the federal level, and of corporate centrality, as well as the presence of different facilities and services.

Normally, the assumption of stationarity of random variables is required to perform many statistical inference methods. For instance, when we want to correlate distinct data sets in order to predict their behaviours the non-stationary in the signals makes standard Pearson correlation coefficient useless in the this hypothetical evaluation. Thus, it is necessary to use statistical models that developing to accommodate the complexity of the data. The detrended cross-correlation (DCCA) method is able to estimate the true correlation coefficient between series precisely ${ }^{16}$. This method is robust to contaminated noises, as daily dengue records, furthermore, the DCCA methods take some advantages in correctly quantifying scale-dependent correlations ${ }^{17}$.

The $\rho_{D C C A}$ cross-correlaion coefficient method used is a proposal of ${ }^{18}$, it is based on the self-affinity theory ${ }^{19}$ and the study of cross-correlation between time series defined by power laws ${ }^{20}$. In this context, there are some new progress about cross-correlations methods families, see ${ }^{21-27}$.

Here we explain how to calculate the $\rho_{D C C A}$ cross-correlation coefficient briefly. In the firs step, consider two time series, $\left\{x_{t}\right\}$ and $\left\{y_{t}\right\}$ where $t=1,2, \ldots, N$ (N is the total number of elements of the time series), it is calculated the random walk profile 


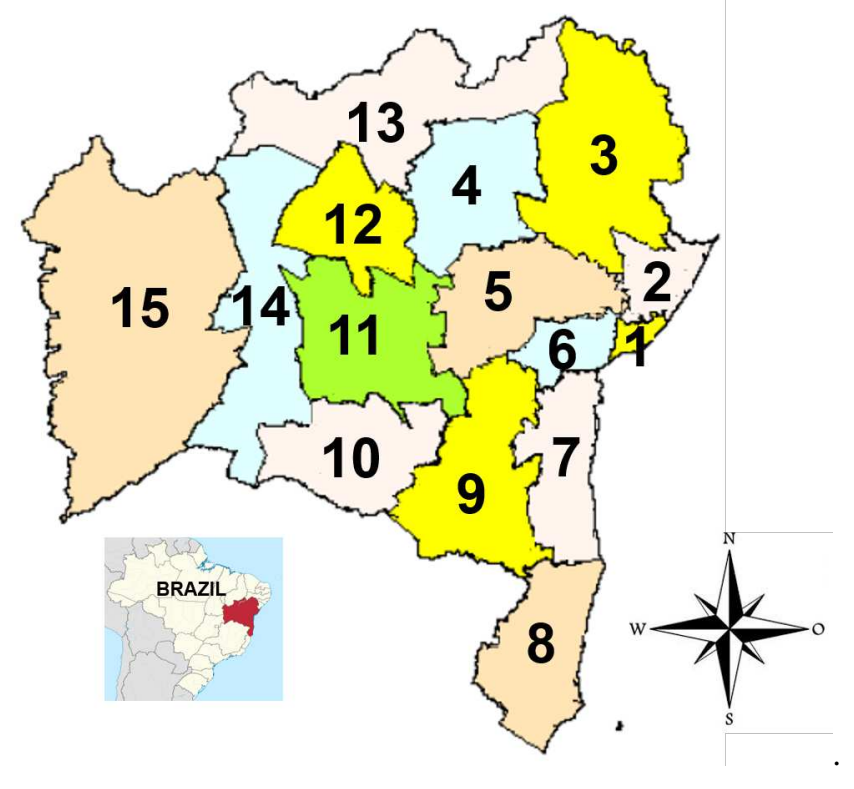

Figure 4. Economic regions in Bahia, Brazil. 1 - Região Metropolitana de Salvador (RMS), 2 - Litoral Norte (LTN), 3 Nordeste (NDE), 4 - Piemonte da Diamantina(PIE), 5 - Paraguaçu (PGU), 6 - Recôncavo (REC), 7 - Litoral Sul (LTS), 8Extremo Sul (EXS), 9 - Sudoeste (SDE), 10 - Serra Geral (SGE), 11 - Chapada Diamantina (CHP), 12 - Irecê (IRC), 13 - Baixo Médio São Francisco (BMF), 14 - Médio São Francisco (MSF), 15 - Oeste (OST). The arrangement was extracted from ${ }^{15}$.

by integrating the original time series obtaining two new series (Equation 1).

$$
x x_{k}=\sum_{t=1}^{k} x_{t} \text { and } y y_{k}=\sum_{t=1}^{k} y_{t}, k=1,2, \ldots, N
$$

Second, these two integrated time series are divided, $\left\{x x_{k}\right\}$ and $\left\{y y_{k}\right\}$, in $(N-s)$, overlapping boxes of equal length $s$, with $4 \leq s \leq \frac{N}{4}$.

The local trend of each box is calculated by the least squares adjustment of each series, $x P_{i}(k)$ and $y P_{i}(k)$. Then, it is calculated the covariance of residuals in each defined box by Equation 2 .

$$
f_{x y}^{2}(s, i)=\frac{1}{s+1} \sum_{k=1}^{i+s}\left(x x_{k}-x P_{i}(k)\right)\left(y y_{k}-y P_{i}(k)\right)
$$

Next, it is calculated the average over all overlapping boxes to obtain the new covariance function (Equation 3).

$$
F_{x y}^{2}(s)=\frac{1}{N-s} \sum_{i=1}^{N-s} f_{x y}^{2}(s, i)
$$

Then, the $\rho_{D C C A}$ is determined by the Equation 4 .

$$
\rho_{D C C A}(s)=\frac{F^{2}{ }_{x y}(s)}{F_{x x}(s) F_{y y}(s)}
$$

Where $F_{x y}^{2}(s)$ is the correlation function determined by the method of ${ }^{20} . F_{x x}(s)$ and $F_{y y}(s)$ are the auto correlation functions determined by the method of ${ }^{19}$. Moreover, the cross-correlation coefficient depends on the size of each box $\mathrm{s}$.

In terms of the level of cross-correlation coefficient $\rho_{D C C A}$ has a range of $[-1,1]$, which 1 equal to a perfect cross-correlation and -1 is a perfect anti cross-correlation. A zero value represents no cross-correlated condition ${ }^{28}$. 

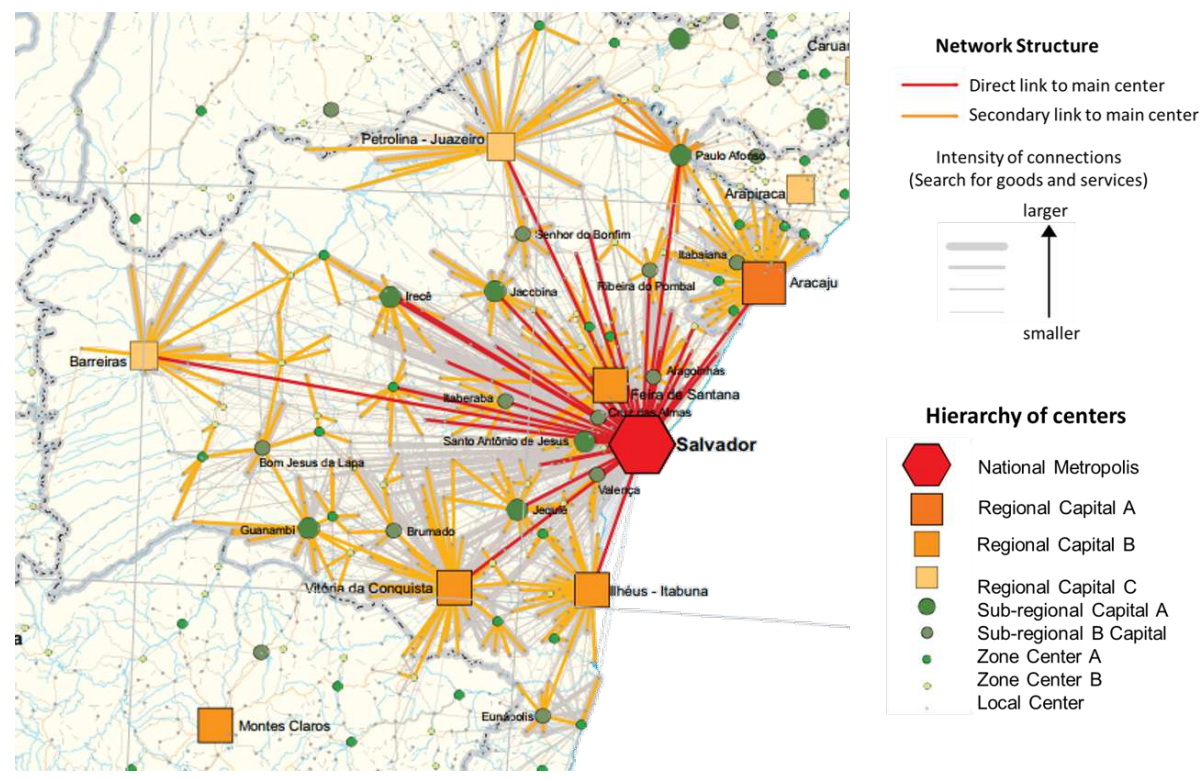

Figure 5. Salvador capital of Bahia, into the RMS is characterized by include intensity of the connections between cities, plays a role of territorial management, evaluating levels of centrality of the executive and judiciary at the federal level, and of corporate centrality, as well as the presence of different facilities and services. Figure adapted from ${ }^{6}$

Furthermore, for the analysis of the results, it was considered the statistical test of ${ }^{29}$ in which the correlation is significant outside the lines representing lower and upper critical values with significance of $95 \%$, for the test with the hypothesis $\mathrm{H} 0: \rho_{D C C A}=0$ and $\mathrm{H} 1: \rho_{D C C A} \neq 0$.

\section{References}

1. Bhatt, S. et al. The global distribution and burden of dengue. Nature 496, 504-507 (2013).

2. Saba, H. et al. Relevance of transportation to correlations among criticality, physical means of propagation, and distribution of dengue fever cases in the state of bahia. Sci. The Total. Environ. (2018).

3. Duncombe, J. et al. Geographical information systems for dengue surveillance. The Am. journal tropical medicine hygiene 86, 753-755 (2012).

4. Kan, C.-C. et al. Two clustering diffusion patterns identified from the 2001-2003 dengue epidemic, kaohsiung, taiwan. The Am. journal tropical medicine hygiene 79, 344-352 (2008).

5. Thammapalo, S., Chongsuvivatwong, V., Geater, A. \& Dueravee, M. Environmental factors and incidence of dengue fever and dengue haemorrhagic fever in an urban area, southern thailand. Epidemiol. \& Infect. 136, 135-143 (2008).

6. IBGE. Instituto brasileiro de geografia e estatística - (ibge). URL: www.ibge.gov.br (2017).

7. Cardoso, H. S. P., Miranda, J. G. V., de Freitas Jorge, E. M. \& Moret, M. A. Correlation between transport and occurrence of dengue cases in bahia. J. Commun. Comput. 11, 217-221 (2014).

8. NOAA. National oceanic and atmospheric administration. Available in: https://www.esrl.noaa.gov/psd/data/gridded/data.south_america_precip.html (2017).

9. Mondini, A. \& Chiaravalloti-Neto, F. Spatial correlation of incidence of dengue with socioeconomic, demographic and environmental variables in a brazilian city. Sci. Total. Environ. 393, 241-248 (2008).

10. Saba, H., Vale, V. C., Moret, M. A. \& Miranda, J. G. V. Spatio-temporal correlation networks of dengue in the state of bahia. BMC public health 14, 1085 (2014).

11. Nascimento Filho, A. et al. Self-affinity and self-organized criticality applied to the relationship between the economic arrangements and the dengue fever spread in bahia. Phys. A: Stat. Mech. its Appl. 502, 619-628 (2018). 
12. da Silva, M. F. et al. Quantifying the contagion effect of the 2008 financial crisis between the $\mathrm{g} 7$ countries (by gdp nominal). Phys. A: Stat. Mech. its Appl. 453, 1-8 (2016).

13. SVS. Secretaria de vigilnâcia sanitária - boletim epidemiólgico-dengue 43, p. 11, 2012 . Available in: http://portalms.saude.gov.br/vigilancia-em-saude/ (2012).

14. Filho, A., Zebende, G. \& Moret, M. Self-affinity of vehicle demand on the ferry-boat system. Int. J. Mod. Phys. C 19, 665-669 (2008).

15. SEI. Superintêndncia, de estudos econômicos e sociais do estado da bahia (sei). regiões econômicas do estado da bahia. Available in: http://www.sei.ba.gov.br/ (2012).

16. Kristoufek, L. Detrending moving-average cross-correlation coefficient: Measuring cross-correlations between nonstationary series. Phys. A: Stat. mechanics its applications 406, 169-175 (2014).

17. Piao, L. \& Fu, Z. Quantifying distinct associations on different temporal scales: comparison of dcca and pearson methods. Sci. reports 6, 36759 (2016).

18. Zebende, G. F. Dcca cross-correlation coefficient: quantifying level of cross-correlation. Phys. A: Stat. Mech. its Appl. 390, 614-618 (2011).

19. Peng, C.-K. et al. Mosaic organization of dna nucleotides. Phys. review e 49, 1685 (1994).

20. Podobnik, B. \& Stanley, H. E. Detrended cross-correlation analysis: a new method for analyzing two nonstationary time series. Phys. review letters 100, 084102 (2008).

21. Jiang, Z.-Q., Zhou, W.-X. et al. Multifractal detrending moving-average cross-correlation analysis. Phys. Rev. E 84, 016106 (2011).

22. Kristoufek, L. Detrending moving-average cross-correlation coefficient: Measuring cross-correlations between nonstationary series. Phys. A: Stat. Mech. its Appl. 406, 169-175 (2014).

23. Kwapień, J., Oświkecimka, P. \& Drożdż, S. Detrended fluctuation analysis made flexible to detect range of cross-correlated fluctuations. Phys. Rev. E 92, 052815 (2015).

24. Qian, X.-Y. et al. Detrended partial cross-correlation analysis of two nonstationary time series influenced by common external forces. Phys. Rev. E 91, 062816 (2015).

25. Wang, G.-J., Xie, C., He, L.-Y. \& Chen, S. Detrended minimum-variance hedge ratio: A new method for hedge ratio at different time scales. Phys. A: Stat. Mech. its Appl. 405, 70-79 (2014).

26. Yuan, N., Xoplaki, E., Zhu, C. \& Luterbacher, J. A novel way to detect correlations on multi-time scales, with temporal evolution and for multi-variables. Sci. reports 6, 27707 (2016).

27. Wang, G.-J., Xie, C., Lin, M. \& Stanley, H. E. Stock market contagion during the global financial crisis: A multiscale approach. Finance Res. Lett. 22, 163-168 (2017).

28. Machado Filho, A., Da Silva, M. \& Zebende, G. Autocorrelation and cross-correlation in time series of homicide and attempted homicide. Phys. A: Stat. Mech. its Appl. 400, 12-19 (2014).

29. Podobnik, B., Jiang, Z.-Q., Zhou, W.-X. \& Stanley, H. E. Statistical tests for power-law cross-correlated processes. Phys. Rev. E 84, 066118 (2011).

\section{Acknowledgements}

This work received financial support from the National Council for Scientific and Technological Development - CNPq, grant numbers 431990/2018-2 , 313423/2019-9 , 305291/2018-1 and 431651/2018-3.

\section{Author contributions statement}

A.F. and T.M conducted the experiment(s), P.F, H.S. and M.M. analysed the results. All authors reviewed the manuscript. 


\section{Figures}

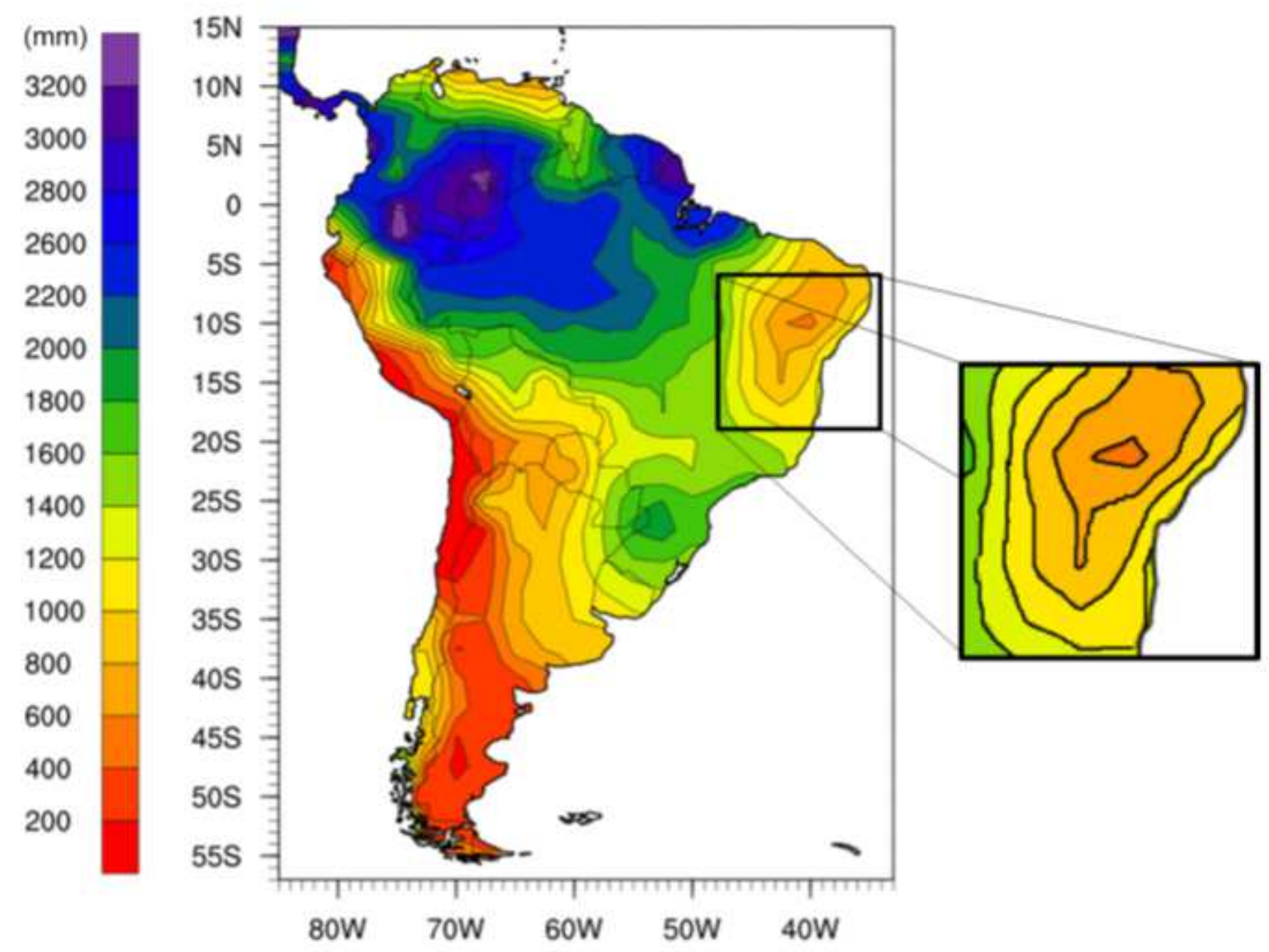

\section{Figure 1}

The annual total precipitation in Bahia. According to the Physical Science Division of U.S. department of commerce between 1976 and 2009 this was less than 1,000mm. Figure adapted from8 Note: The designations employed and the presentation of the material on this map do not imply the expression of any opinion whatsoever on the part of Research Square concerning the legal status of any country, territory, city or area or of its authorities, or concerning the delimitation of its frontiers or boundaries. This map has been provided by the authors. 


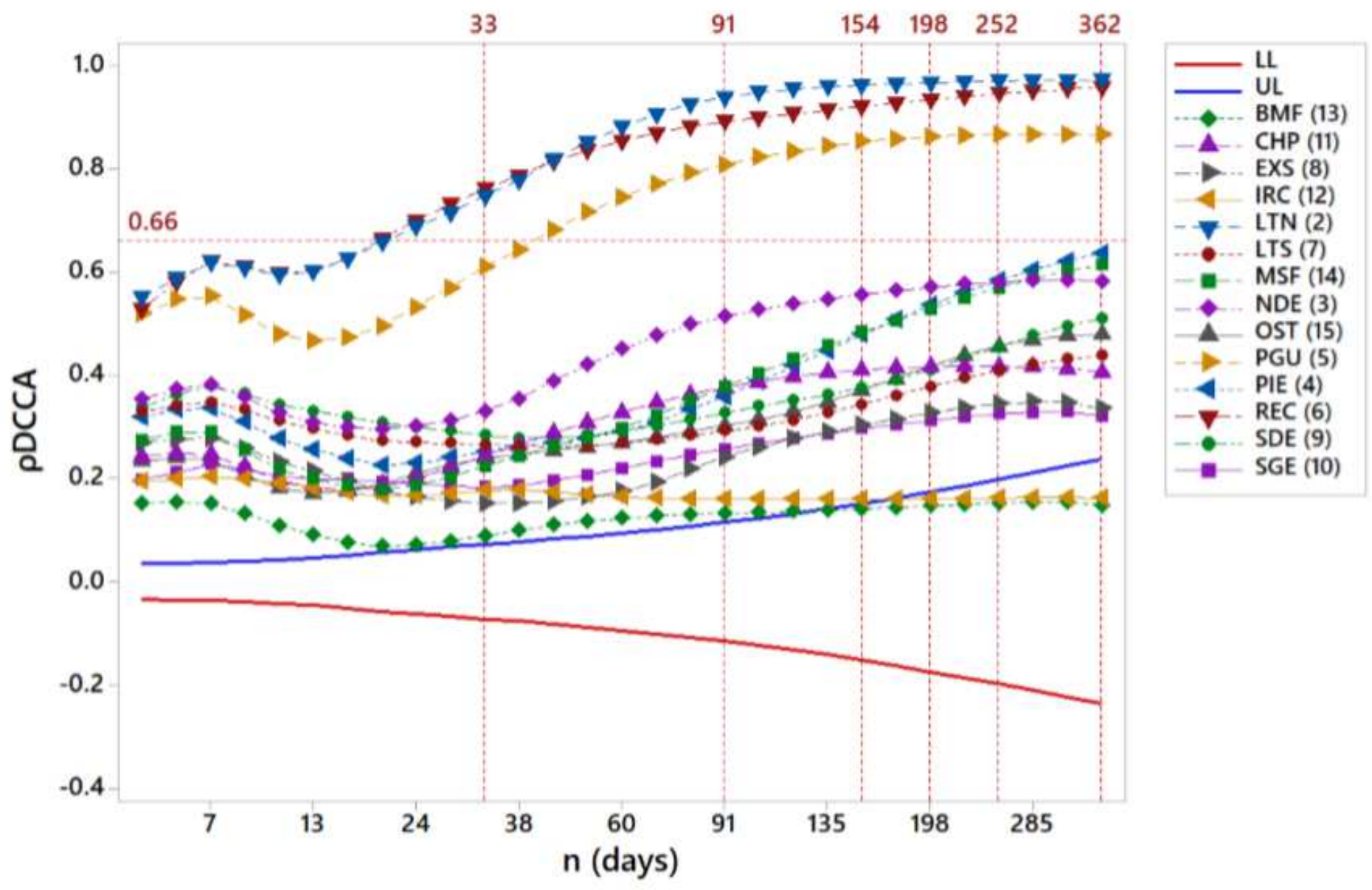

Figure 2

Cross-correlation results for 29 different time scales. The values forpDCCA between RMS and the nearby ER presented a strong significant correlation. LTN, REC and PGU stood out by exceeding the correlation level of 0.66 , from the scale of 33 days, remaining strong for all subsequent time scales. $95 \% \mathrm{LL}$ and $95 \% \mathrm{UL}$ represent the lower and upper limits to consider for the test of the existence or not of significant correlation 

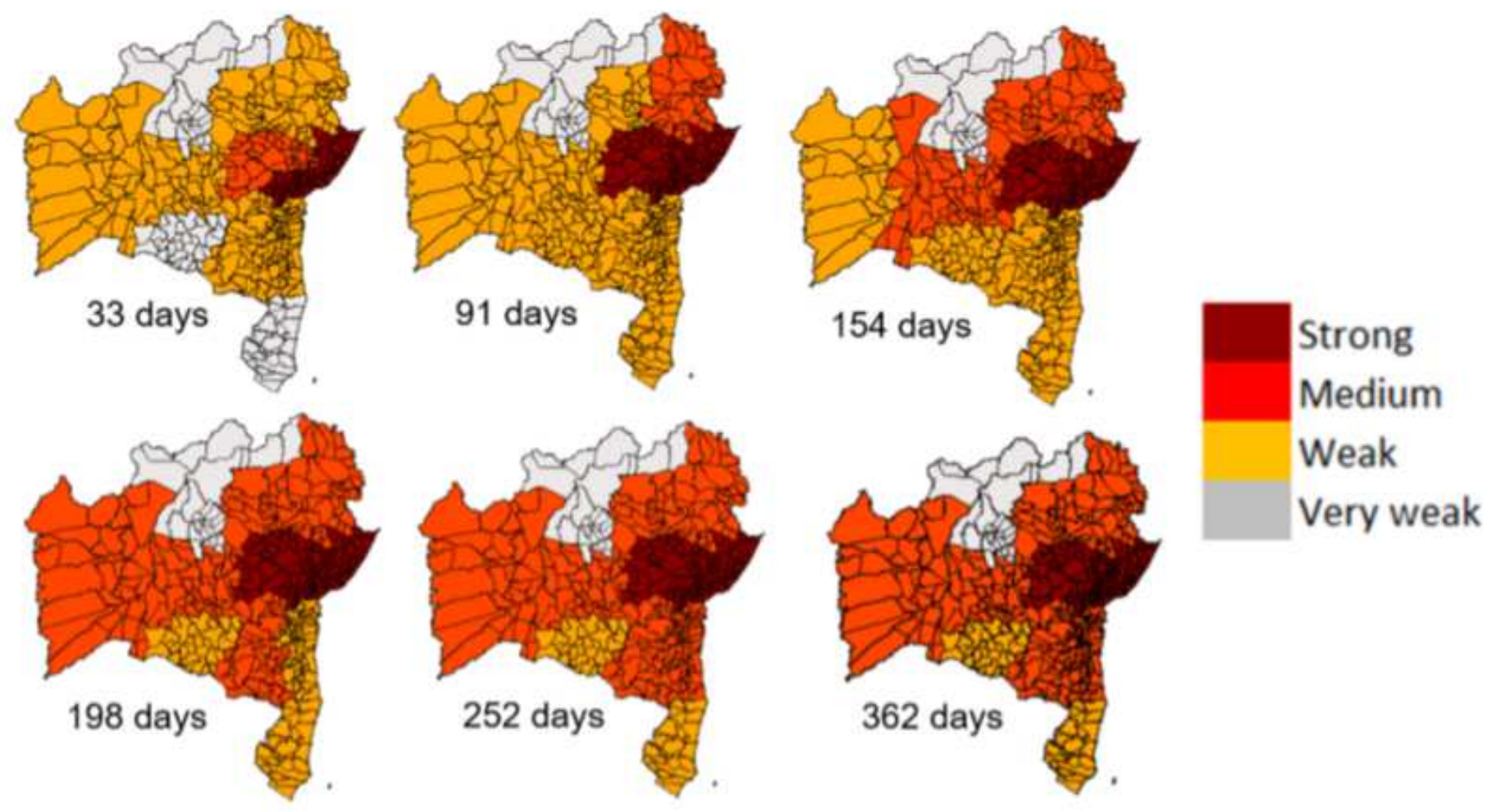

\section{Figure 3}

Correlation degree between RMS and all economic regions in Bahia-Brazil for different time scales. The value of thepDCCA between RMS and all ER presented a significant correlation. This was calculated for six scale days, 33, 91, 154, 198, 252 and 362 days. ThepDCCA's ranges were associated with colors (Strong: $\rho D C C A>0.66$, Medium: $0.4<\rho D C C A \leq 0.66$, Weak: $0.2<\rho D C C A \leq 0.4$, and Very weak: $\rho D C C A \leq 0.2$ ) in order assist understanding. Note: The designations employed and the presentation of the material on this map do not imply the expression of any opinion whatsoever on the part of Research Square concerning the legal status of any country, territory, city or area or of its authorities, or concerning the delimitation of its frontiers or boundaries. This map has been provided by the authors. 


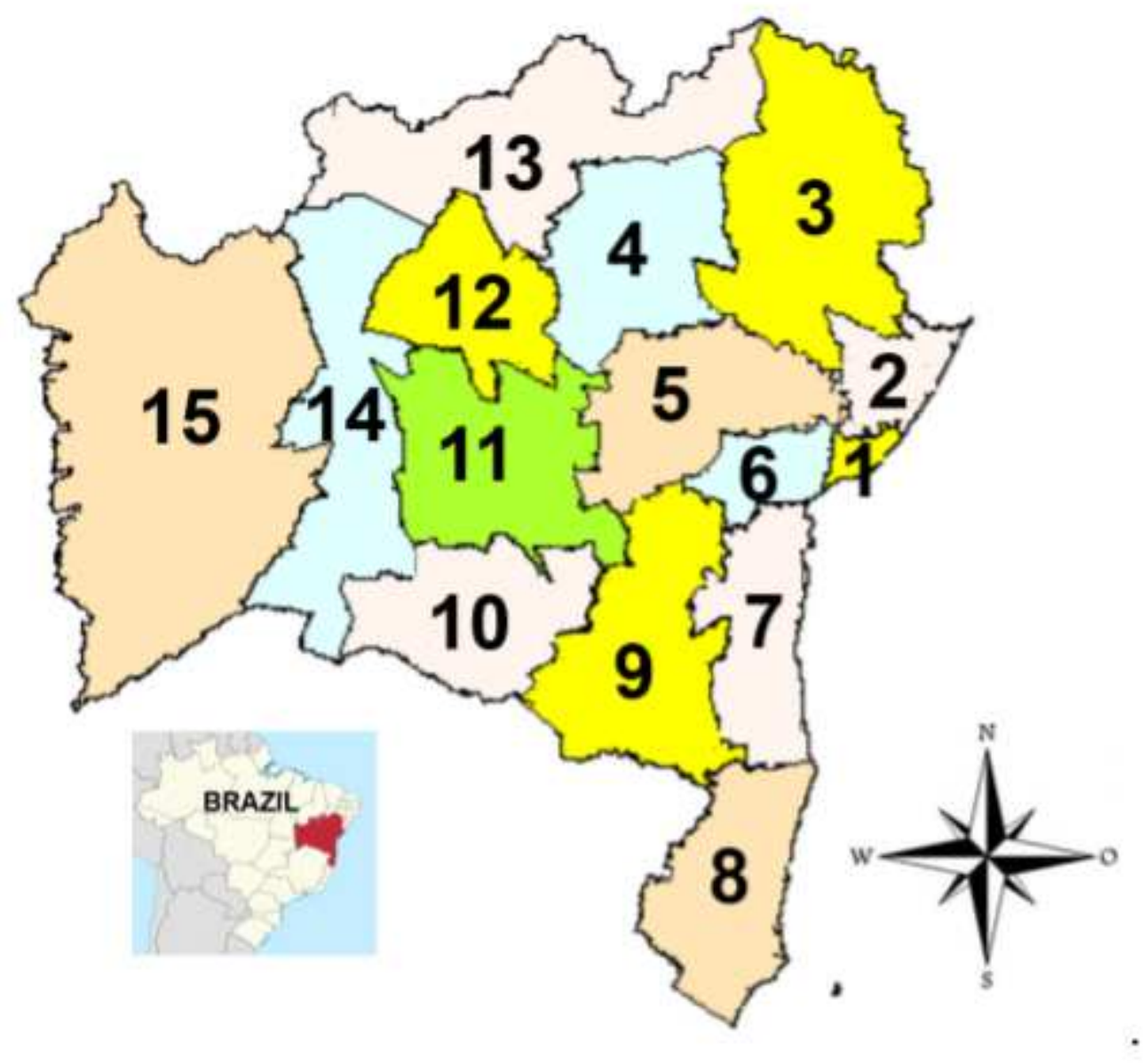

Figure 4

Economic regions in Bahia, Brazil. 1 - Região Metropolitana de Salvador (RMS), 2 - Litoral Norte (LTN), 3 Nordeste (NDE), 4 - Piemonte da Diamantina(PIE), 5 - Paraguaçu (PGU), 6 - Recôncavo (REC), 7 - Litoral Sul (LTS), 8ExtremoSul(EXS), 9-Sudoeste(SDE),10-SerraGeral(SGE),11-ChapadaDiamantina(CHP),12Irecê(IRC),13-Baixo Médio São Francisco (BMF), 14 - Médio São Francisco (MSF), 15 - Oeste (OST). The arrangement was extracted from15. Note: The designations employed and the presentation of the material on this map do not imply the expression of any opinion whatsoever on the part of Research Square concerning the legal status of any country, territory, city or area or of its authorities, or concerning the delimitation of its frontiers or boundaries. This map has been provided by the authors. 


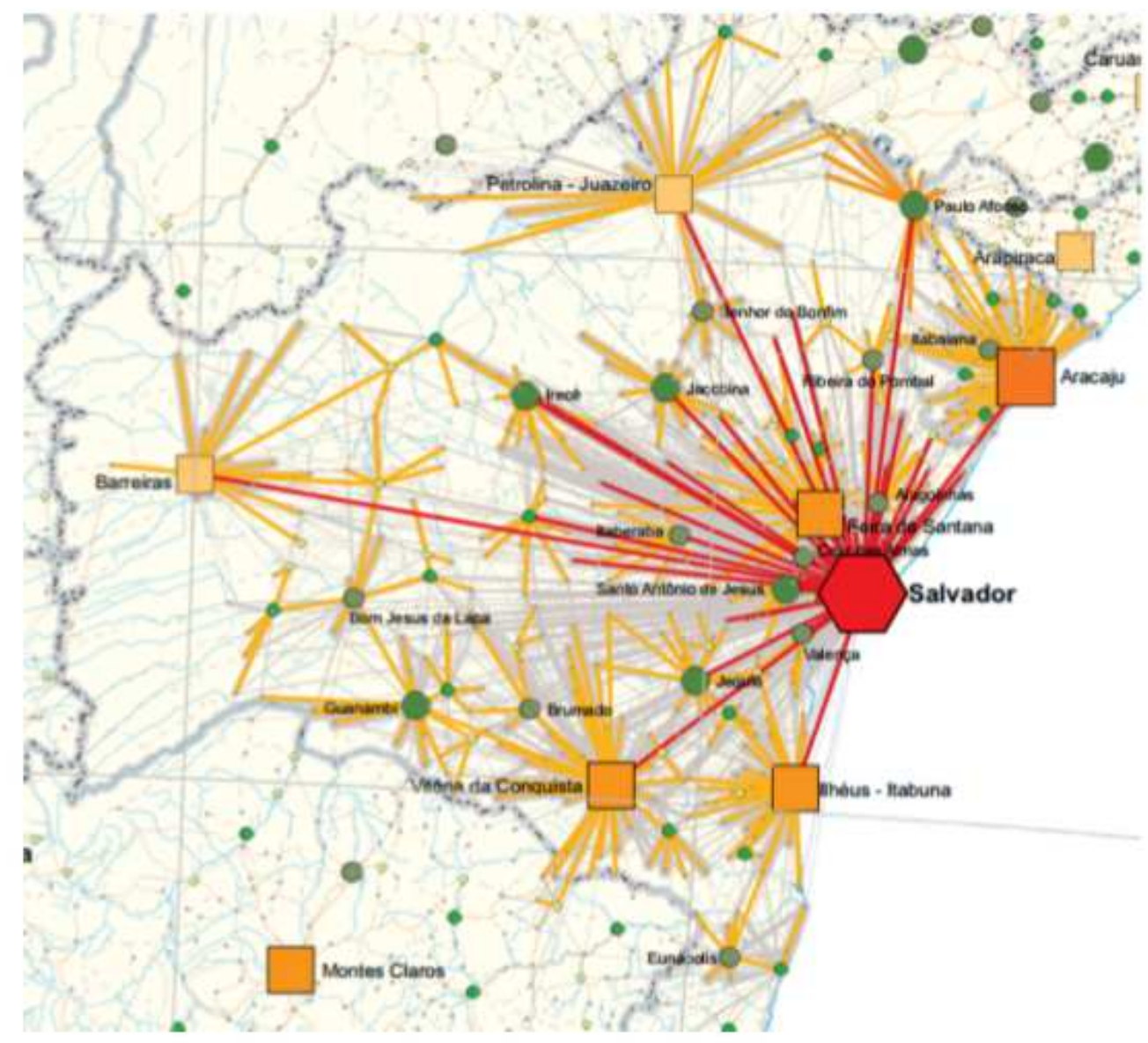

\section{Network Structure}

Direct link to main center Secondary link to main center

Intensity of connections (Search for goods and services)

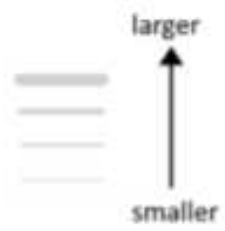

Hierarchy of centers

National Metropolis

Regional Capital A

Regional Capital B

Regional Capital C Sub-regional Capital A Sub-regional B Capital Zone Center $A$ Zone Center B Local Center

\section{Figure 5}

alvador capital of Bahia, into the RMS is characterized by include intensity of the connections between cities, plays a role of territorial management, evaluating levels of centrality of the executive and judiciary at the federal level, and of corporate centrality, as well as the presence of different facilities and services. Figure adapted from6 Note: The designations employed and the presentation of the material on this map do not imply the expression of any opinion whatsoever on the part of Research Square concerning the legal status of any country, territory, city or area or of its authorities, or concerning the delimitation of its frontiers or boundaries. This map has been provided by the authors. 